\title{
Use of crop condition based dummy regressor and weather input for parameter estimation of mustard yield forecast models
}

\author{
Ravita* $^{*}$ and Urmil Verma \\ *Department of Mathematics, Statistics and Physics, CCS Haryana Agricultural University, Hisar-125004, INDIA \\ Corresponding author. E-mail: vermas21@hotmail.com
}

Received: October 6, 2016; Revised received: April 9, 2017; Accepted: August 13, 2017

\begin{abstract}
Parameter estimation in statistical modelling plays a crucial role in the real world phenomena. Several alternative analyses may be required for the purpose. In this paper, standard linear regression and multivariate statistical analyses were carried out to achieve the district-level rapeseed-mustard yield estimation in Haryana State (India). The study revealed that the zonal weather models incorporating crop condition term as dummy regressor(s) had the desired predictive accuracy. The model based mustard yield(s) indicated good agreement with State Department of Agriculture (DOA) yield estimates by showing 5-10 percent deviations in most of the mustard growing districts however for two-three districts, it gave 12-13 percent deviations possibly due to the smaller set of data available for those districts. The crop yield estimates on the basis of developed models may be obtained 4-5 weeks in advance of the harvest time.
\end{abstract}

Keywords: Clustering, Dummy variables, Linear time trend, Multiple linear regression, Principal component scores,

\section{INTRODUCTION}

Climate change is a major concern these days, and the researchers are engaged in understanding its impact on growth and yield of crops, and also identifying suitable management options to sustain the productivity of crops under projected climate change scenario. Changes in seasonal temperatures affect the crop yield, mainly through their effect on phenological and developmental processes. Winter crops are especially vulnerable to high temperatures during the reproductive stages and their differential responses to rising temperatures can have important consequences for crop yield. Hence, an understanding of the responses of crops to seasonality in temperature and fluctuations in other components of the weather is basic in evaluating and anticipating the impacts of the projected future climatic scenario on crop yields. In many previous studies, yield forecasting models have incorporated a series of weather predictors (Kandiannan et al., 2002, Dadhwal et al., 2003, Andarzian et al., 2008, Ahmad and Kathuria, 2010 and Adrian, 2012 ). Shabnam et al. (2013) have used time series data of temperature and yield to assess the impact of climate change on mustard crop yield in Haryana. Verma et al. (2011) and Goyal and Verma (2015) have used agromet/spectral indices in context of pre-harvest yield forecasting of different crops in Haryana. Azfar et al. (2015) carried out principal component analysis for rapeseed and mustard yield forecast models for Faizabad district of U.P. (India).
An attempt in this study has been made to develop zonal weather-yield models for major mustard growing districts; Mahendergarh, Rewari, Jhajjar, Gurgaon, Hisar, Bhiwani, Sirsa and Fatehabad (more than 90\% crop covered) of Haryana with the objective of selection of weather variables along with (or without) crop condition based categorical covariate by following multiple linear regression and principal component analyses.

\section{MATERIALS AND METHODS}

Crop status and data description: Rapeseed-mustard is the second most important edible oilseed after groundnut sharing $27.8 \%$ in the India's oilseed economy. The global production of rapeseed-mustard and its oil is around 38-42 and 12-14 mt, respectively. India is the fourth largest oilseed economy in the world and it contributes $28.3 \%$ and $19.8 \%$ in world acreage and production. Indian mustard (Brassica juncea (L.) Czern \& Coss.) is predominantly cultivated in Rajasthan, UP, Haryana, Madhya Pradesh and Gujarat. It is basically a winter crop and is grown in the rabi season from September-October to February-March. The crop grows well in areas receiving 25 to $40 \mathrm{~cm}$ of rainfall and this is provided by the monsoon rains during the sowing season of the crop in India. The rapeseed and mustard thrive best in light to heavy loamy soils.

The Haryana state comprising of 21 districts (geographical area: $4.42 \mathrm{~m} \mathrm{ha}$ ) is situated between $74^{\circ} 25^{\prime}$ to $77^{\circ} 38^{\prime} \mathrm{E}$ longitude and $27^{\circ} 40^{\prime}$ to $30^{\circ} 55^{\prime} \mathrm{N}$ latitude. A time-series of state Department 
of Agriculture (DOA) mustard yield spanning 1966-67 to 2013-14 (Source: esaharyana.gov.in/ State StatisticalAbstract/) and weather data from 1980-81 to 201314 were collected from different meteorological observatories in Haryana.. The major mustard growing districts; Hisar, Bhiwani, Sirsa, Fatehabad, Mahendergarh, Rewari, Jhajjar and Gurgaon (more than $90 \%$ of the crop covered) were grouped into a single zone for model building. The zonal models have been fitted using the mustard yield data for the period 1966-67 to 2008-09 of Hisar, Mahendergarh and Gurgaon districts and 1972-73 to 2008-09 of Bhiwani district, 1975-76 to 2008-09 of Sirsa district and 199798 to 2008-09 of Fatehabad and Jhajjar districts. However, the fortnightly weather data have been utilized from 1980-81 to 2008-09. The predictive performance(s) of the models developed under the alternative analyses have been comparatively evaluated for the post-sample period(s) i.e. $2009-10$ to 2013-14.

Statistical methodology adopted: The weather variables affect the crop differentially during different phases of its growth period. Thus, to integrate the weather variables over different growth phases, the crop growth period (September to February) was divided into 12 fortnights and daily weather data summarized on a fortnightly basis for the period 198081 to 2008-09 were used for the model building. The linear time-trend model(s) i.e. $\mathrm{T}_{\mathrm{r}}=\mathrm{a}+\mathrm{br}$, where $\mathrm{T}_{\mathrm{r}}=$ Yield ( $\mathrm{q} / \mathrm{ha}), \mathrm{a}=$ Intercept, $\mathrm{b}=$ Slope and $\mathrm{r}=$ Year were fitted on the basis of time-series crop yield data to obtain the predictions $\mathrm{T}_{\mathrm{r}}$ and that has been used as 'trend yield'.

Multiple linear regression based zonal weather-yield models: The multiple linear regression model defined over the crop growth period containing average maximum temperature, average minimum temperature, average relative humidity (morning + evening) and average sunshine hours for 10 fortnights covering the period October to February and accumulated rainfall for 12 fortnights over the period September to February (Verma et al., 2011) may be expressed as follows:

where,

$\mathrm{Y}=$ yield $(\mathrm{q} / \mathrm{ha}) ; \mathrm{a}_{0}=$ Overall mean effect; $Y=a_{o}+\sum_{i=1}^{10} b_{i} T M X_{i}+\sum_{j=1}^{10} b_{j} T M N_{j}+\sum_{k=1}^{12} b_{k} A R F_{k}+\sum_{l=1}^{10} b_{l} R H_{l}+\sum_{m=1}^{10} b_{m} S S H_{m}+c T_{r}+e$

$b_{i}, b_{j}, b_{k,}, b_{1}, b_{m}=$ Regression coefficients of the weather variables; $\mathrm{i}, \mathrm{j}, \mathrm{k}, \mathrm{l}, \mathrm{m}=$ Meteorological fortnights $(1,2,3 \ldots$ $10 / 12) ; c=$ Regression coefficient of trend yield; $\mathrm{T}_{\mathrm{r}}=$ Trend yield $(\mathrm{q} / \mathrm{ha})$; $\mathrm{e}=$ Error term with the assumption $\operatorname{NID}\left(0, \sigma^{2}\right)$

Principal component analysis: Principal component analysis (PCA) offers considerable improvement over the least squares method in the presence of multicollinearity. It consists of finding the eigen roots and eigen vectors of the correlation matrix of explanatory variables. The most frequently used convention is to retain the components whose eigen values are greater than one. Kaiser (1960) suggested the dropping of components having eigen roots less than 1 . The results of PCA are usually discussed in terms of component scores (the transformed variable values corresponding to a particular case in the data) and loadings (the variance each original variable would have if the data were projected onto a given PCA axis) (Shaw, 2003). The component scores can be used as new variables in multiple regression analysis, while the component loadings are especially useful in determining the substantive importance of a particular variable to a component (Field, 2000).

Considering the standard linear regression model $\mathrm{Y}=$ $\mathrm{X} \mathrm{b}+\hat{\mathrm{I}}$; the values of the PCs for each variable may be given by $Z=X A$ where $(i, j)^{\text {th }}$ element of $Z$ is the value of $i^{\text {th }} P C$ for $j^{\text {th }}$ observation and $A$ is a (pxp) orthogonal matrix whose columns are the eigen vectors associated with $\lambda_{1}, \lambda_{2} \ldots \lambda_{\mathrm{p}}$. Since $\mathrm{A}$ is an orthogonal matrix, $\mathrm{X} b$ can be written as $X \mathrm{AA}^{\prime} \quad \mathrm{b}=\mathrm{Z} v$ where $v$ $=A^{\prime} b$. Thus $Y=Z v+\in$; this has simply replaced the predictor variables by their PCs in the regression model. To overcome the problem of multicollinearity observed among weather variables; multiple linear regression (stepwise method) was again followed by taking DOA yield as the dependent variable and fortnightly weather scores (or PC scores) along with trend yield as independent variables (Draper and Smith, 1981).

Using crop condition based categorical covariate/ dummy variables in multiple linear regression analysis: In many practical situations, time trends occur in response data. Sometimes the trend is the only factor affecting the response and sometimes the time trend effect occurs in addition to the effects produced by other predictor variables. This may be an advantage if one can use it to exploit some special feature of the data. Thus, an indicator variable may be used as a substitute for a quantitative regressor with different allocated codes (Draper and Smith, 1981). An advantage of the indicator variable approach is that it does not require the analyst to make any prior assumption(s) about the functional form of the relationship between response and predictor variable(s). Once again, the zonal weather-yield models were fitted by taking dummy variables along with weather input as regressors and DOA mustard yield as regressand.

\section{RESULTS AND DISCUSSION}

The objective of this empirical study was to assess the predictive accuracies of the developed models for estimating crop yields and how the accuracies are influenced under alternative analyses. Initially, the best 
Ravita and Urmil Verma / J. Appl. \& Nat. Sci. 9 (3): 1703- 1709 (2017)

Table 1. Principal component matrix alongwith variance explained by different components.

\begin{tabular}{|c|c|c|c|c|c|c|c|c|c|c|c|c|c|c|}
\hline \multirow{2}{*}{$\begin{array}{l}\text { Weather } \\
\text { parameters }\end{array}$} & \multicolumn{14}{|c|}{ Component(s) } \\
\hline & 1 & 2 & 3 & 4 & 5 & 6 & 7 & 8 & 9 & 10 & 11 & 12 & 13 & 14 \\
\hline $\mathrm{TMX}_{1}$ & 0.76 & -0.01 & 0.10 & 0.12 & 0.28 & 0.02 & -0.10 & 0.02 & -0.13 & 0.02 & 0.29 & -0.03 & -0.11 & -0.18 \\
\hline $\mathrm{TMX}_{2}$ & 0.82 & -0.07 & 0.03 & -0.08 & -0.05 & 0.06 & -0.09 & -0.16 & -0.07 & -0.10 & 0.06 & -0.01 & -0.34 & -0.15 \\
\hline $\mathrm{TMX}_{3}$ & 0.45 & 0.10 & 0.20 & -0.20 & 0.01 & 0.03 & -0.06 & 0.14 & -0.39 & -0.13 & -0.06 & 0.01 & -0.12 & -0.10 \\
\hline $\mathrm{TMX}_{4}$ & 0.55 & 0.11 & -0.13 & -0.23 & -0.01 & -0.09 & -0.26 & 0.22 & 0.09 & 0.27 & 0.20 & -0.43 & 0.09 & -0.22 \\
\hline $\mathrm{TMX}_{5}$ & 0.65 & -0.04 & -0.26 & 0.13 & -0.13 & 0.26 & -0.12 & 0.24 & 0.10 & 0.40 & -0.24 & -0.14 & -0.03 & 0.00 \\
\hline $\mathrm{TMX}_{6}$ & 0.74 & 0.11 & 0.15 & -0.46 & -0.05 & -0.02 & -0.07 & 0.00 & -0.01 & 0.07 & 0.03 & 0.09 & 0.15 & -0.25 \\
\hline $\mathrm{TMX}_{7}$ & -0.03 & -0.04 & 0.04 & -0.01 & -0.15 & -0.85 & 0.08 & 0.10 & -0.21 & 0.14 & 0.08 & -0.12 & -0.01 & 0.01 \\
\hline $\mathrm{TMX}_{8}$ & 0.33 & 0.08 & 0.69 & 0.49 & 0.02 & -0.19 & -0.06 & 0.03 & 0.03 & -0.18 & 0.04 & 0.08 & 0.04 & -0.14 \\
\hline $\mathrm{TMX}_{9}$ & -0.04 & -0.35 & 0.30 & 0.35 & -0.18 & -0.08 & -0.12 & 0.24 & -0.21 & 0.12 & 0.17 & 0.45 & 0.38 & -0.21 \\
\hline $\mathrm{TMX}_{10}$ & 0.13 & -0.53 & -0.35 & 0.22 & 0.29 & -0.04 & -0.24 & -0.19 & -0.32 & -0.25 & -0.00 & 0.15 & 0.07 & -0.16 \\
\hline $\mathrm{TMN}_{1}$ & -0.03 & 0.07 & 0.02 & 0.07 & 0.04 & 0.04 & -0.09 & 0.91 & 0.12 & -0.08 & -0.04 & -0.03 & -0.02 & -0.06 \\
\hline $\mathrm{TMN}_{2}$ & -0.04 & 0.11 & -0.22 & 0.14 & 0.67 & 0.04 & 0.01 & 0.49 & 0.19 & 0.05 & -0.11 & -0.06 & -0.06 & -0.08 \\
\hline $\mathrm{TMN}_{3}$ & 0.05 & 0.01 & -0.07 & 0.21 & 0.75 & 0.20 & 0.20 & 0.03 & -0.06 & -0.06 & -0.06 & -0.06 & 0.04 & 0.24 \\
\hline $\mathrm{TMN}_{4}$ & -0.07 & 0.25 & 0.30 & -0.04 & 0.18 & -0.35 & 0.06 & 0.33 & 0.35 & 0.49 & 0.02 & 0.09 & -0.02 & -0.05 \\
\hline $\mathrm{TMN}_{5}$ & -0.35 & 0.04 & -0.18 & 0.11 & 0.04 & -0.04 & -0.05 & -0.06 & 0.04 & 0.11 & 0.03 & 0.05 & -0.18 & 0.84 \\
\hline $\mathrm{TMN}_{6}$ & -0.09 & 0.19 & 0.05 & 0.14 & -0.07 & 0.16 & -0.02 & 0.11 & 0.82 & 0.06 & -0.22 & 0.02 & 0.08 & 0.14 \\
\hline $\mathrm{TMN}_{7}$ & -0.09 & -0.16 & -0.05 & 0.14 & 0.06 & 0.05 & 0.15 & 0.03 & -0.16 & 0.77 & 0.02 & -0.25 & -0.12 & $0 . .27$ \\
\hline $\mathrm{TMN}_{8}$ & 0.19 & 0.12 & -0.28 & 0.81 & 0.13 & 0.02 & 0.07 & 0.10 & 0.17 & 0.04 & -0.06 & -0.01 & -0.10 & 0.16 \\
\hline $\mathrm{TMN}_{9}$ & -0.18 & 0.53 & 0.20 & 0.44 & -0.14 & -0.16 & 0.11 & 0.29 & 0.02 & 0.31 & 0.04 & 0.17 & 0.25 & -0.12 \\
\hline $\mathrm{TMN}_{10}$ & -0.01 & -0.24 & -0.06 & 0.57 & 0.26 & 0.18 & 0.03 & 0.18 & 0.00 & -0.09 & 0.47 & 0.17 & 0.24 & 0.06 \\
\hline $\mathrm{RH}_{1}$ & -0.73 & -0.04 & -0.25 & -0.08 & -0.18 & 0.09 & 0.07 & 0.41 & 0.18 & -0.17 & -0.19 & 0.07 & 0.08 & -0.03 \\
\hline $\mathrm{RH}_{2}$ & -0.82 & 0.14 & -0.17 & 0.01 & 0.29 & 0.06 & 0.07 & 0.17 & 0.10 & -0.07 & -0.01 & 0.10 & 0.23 & 0.08 \\
\hline $\mathrm{RH}_{3}$ & -0.41 & 0.01 & -0.20 & 0.03 & 0.36 & 0.06 & 0.45 & -0.04 & 0.31 & 0.14 & 0.14 & 0.40 & 0.34 & 0.10 \\
\hline $\mathrm{RH}_{4}$ & -0.41 & 0.30 & 0.08 & -0.09 & 0.20 & -0.05 & 0.01 & -0.05 & 0.23 & 0.00 & 0.01 & 0.70 & 0.09 & 0.10 \\
\hline $\mathrm{RH}_{5}$ & -0.73 & 0.14 & -0.04 & 0.17 & 0.11 & -0.02 & -.073 & -0.20 & 0.06 & -0.08 & 0.17 & 0.26 & 0.03 & 0.39 \\
\hline $\mathrm{RH}_{6}$ & -0.59 & 0.10 & -0.22 & 0.57 & 0.10 & 0.22 & -0.02 & 0.05 & 0.27 & -0.11 & -0.10 & -0.12 & -0.14 & 0.14 \\
\hline $\mathrm{RH}_{7}$ & 0.02 & -0.05 & -0.27 & 0.11 & 0.08 & 0.90 & 0.02 & 0.13 & -0.02 & 0.11 & 0.01 & -0.09 & -0.17 & -0.01 \\
\hline $\mathrm{RH}_{8}$ & -0.06 & 0.05 & -0.80 & .255 & 0.11 & 0.40 & 0.03 & 0.17 & -0.02 & -0.06 & 0.02 & 0.00 & -0.11 & 0.17 \\
\hline $\mathrm{RH}_{9}$ & -0.04 & 0.88 & -0.21 & 0.14 & 0.20 & 0.15 & 0.11 & 0.04 & 0.01 & -0.17 & 0.06 & -0.01 & -0.05 & 0.08 \\
\hline $\mathrm{RH}_{10}$ & -0.19 & 0.59 & 0.12 & 0.44 & -0.01 & 0.19 & -0.01 & 0.28 & 0.01 & -0.21 & 0.34 & 0.02 & 0.09 & 0.05 \\
\hline $\mathrm{SSH}_{1}$ & 0.75 & -0.11 & 0.30 & 0.05 & 0.05 & -0.01 & 0.11 & -0.26 & -0.10 & -0.07 & 0.06 & 0.08 & 0.15 & 0.14 \\
\hline $\mathrm{SSH}_{2}$ & 0.64 & -0.38 & 0.20 & 0.06 & -0.51 & -0.08 & 0.16 & -0.13 & 0.05 & 0.01 & -0.05 & -0.08 & -0.04 & 0.19 \\
\hline $\mathrm{SSH}_{3}$ & 0.25 & -0.24 & 0.13 & 0.05 & -0.82 & -0.13 & -0.12 & 0.14 & 0.00 & 0.07 & -0.01 & -0.25 & -0.04 & 0.09 \\
\hline $\mathrm{SSH}_{4}$ & 0.68 & -0.22 & -0.07 & 0.12 & -0.18 & 0.02 & 0.16 & 0.14 & 0.12 & -0.24 & -0.04 & -0.36 & 0.19 & 0.11 \\
\hline $\mathrm{SSH}_{5}$ & 0.69 & -0.22 & -0.12 & 0.26 & -0.36 & 0.13 & 0.23 & 0.17 & 0.17 & -0.09 & -0.17 & -0.08 & 0.05 & -0.20 \\
\hline $\mathrm{SSH}_{6}$ & 0.65 & -0.10 & 0.21 & -0.55 & -0.02 & -0.22 & 0.14 & 0.03 & -0.26 & -0.08 & 0.14 & 0.15 & 0.06 & 0.02 \\
\hline $\mathrm{SSH}_{7}$ & 0.04 & 0.00 & 0.19 & 0.07 & -0.18 & -0.70 & 0.18 & -0.12 & 0.38 & -0.42 & -0.07 & 0.13 & -0.03 & 0.04 \\
\hline $\mathrm{SSH}_{8}$ & 0.23 & -0.16 & 0.87 & -0.15 & -0.21 & -0.15 & -0.00 & 0.08 & -0.02 & 0.01 & -0.01 & 0.00 & -0.06 & 0.01 \\
\hline $\mathrm{SSH}_{9}$ & 0.12 & -0.82 & 0.05 & 0.01 & -0.20 & 0.05 & -0.11 & 0.19 & 0.12 & -0.13 & 0.04 & 0.13 & 0.19 & 0.06 \\
\hline $\mathrm{SSH}_{10}$ & 0.11 & -0.21 & -0.14 & -0.45 & -0.07 & 0.10 & -0.16 & 0.23 & -0.25 & -0.24 & -0.36 & 0.19 & -0.24 & 0.15 \\
\hline $\mathrm{ARF}_{1}$ & 0.11 & -0.12 & 0.14 & -0.03 & 0.06 & -0.13 & -0.13 & -0.07 & -0.09 & -0.11 & 0.02 & 0.04 & 0.87 & -0.15 \\
\hline $\mathrm{ARF}_{2}$ & -0.12 & -0.18 & 0.06 & 0.04 & 0.12 & 0.13 & 0.03 & 0.23 & 0.07 & 0.09 & -0.83 & 0.05 & -0.00 & -0.00 \\
\hline $\mathrm{ARF}_{3}$ & -0.90 & 0.08 & 0.01 & -0.07 & -0.06 & -0.04 & 0.05 & 0.15 & 0.06 & 0.14 & 0.07 & 0.07 & -0.19 & -0.15 \\
\hline
\end{tabular}


Table 1. Contd.

\begin{tabular}{|c|c|c|c|c|c|c|c|c|c|c|c|c|c|c|}
\hline $\mathrm{ARF}_{4}$ & -0.39 & -0.09 & -0.24 & 0.19 & 0.35 & 0.31 & 0.07 & 0.38 & -0.01 & 0.26 & -0.14 & -0.33 & 0.23 & -0.02 \\
\hline $\mathrm{ARF}_{5}$ & 0.12 & 0.08 & -0.06 & -0.01 & 0.07 & -0.07 & 0.96 & -0.03 & -0.02 & 0.04 & -0.00 & -0.06 & -0.00 & -0.07 \\
\hline $\mathrm{ARF}_{6}$ & -0.02 & 0.13 & 0.11 & 0.01 & 0.10 & -0.07 & 0.91 & -0.01 & -0.08 & -0.00 & -0.06 & 0.05 & -0.17 & 0.00 \\
\hline $\mathrm{ARF}_{7}$ & -0.85 & -0.13 & 0.14 & 0.02 & 0.09 & 0.02 & 0.03 & -0.23 & -0.06 & -0.13 & 0.14 & -0.03 & -0.17 & 0.11 \\
\hline $\mathrm{ARF}_{8}$ & -0.12 & -0.11 & 0.05 & 0.14 & 0.10 & -0.09 & 0.01 & 0.10 & 0.81 & -0.10 & 0.24 & 0.09 & -0.19 & -0.12 \\
\hline $\mathrm{ARF}_{9}$ & 0.17 & 0.01 & -0.01 & -0.10 & -0.15 & 0.09 & -0.01 & -0.18 & 0.06 & 0.75 & -0.12 & 0.19 & -0.01 & -0.08 \\
\hline $\mathrm{ARF}_{10}$ & -0.06 & -0.11 & -0.56 & 0.27 & 0.16 & 0.01 & -0.01 & 0.44 & -0.17 & 0.04 & -0.08 & -0.08 & -0.24 & 0.13 \\
\hline $\mathrm{ARF}_{11}$ & -0.04 & 0.82 & -0.01 & -0.05 & 0.01 & -0.14 & -0.05 & 0.06 & 0.13 & -0.04 & 0.04 & 0.27 & 0.01 & -0.00 \\
\hline $\mathrm{ARF}_{12}$ & -0.13 & -0.06 & 0.50 & 0.05 & -0.06 & 0.16 & -0.06 & 0.31 & 0.19 & 0.02 & 0.60 & 0.18 & -0.03 & 0.11 \\
\hline Eigen value & 9.81 & 4.08 & 3.83 & 3.46 & 3.21 & 2.99 & 2.93 & 2.81 & 2.66 & 2.54 & 2.11 & 1.94 & 1.92 & 1.67 \\
\hline $\begin{array}{l}\text { Percent } \\
\text { variance } \\
\text { explained }\end{array}$ & 18.87 & 7.85 & 7.36 & 6.66 & 6.18 & 5.76 & 5.62 & 5.41 & 5.11 & 4.89 & 4.05 & 3.72 & 3.69 & 3.21 \\
\hline $\begin{array}{l}\text { Cumulative } \\
\text { percentage of } \\
\text { total variance }\end{array}$ & 18.87 & 26.72 & 34.07 & 40.73 & 46.91 & 52.67 & 58.29 & 63.70 & 68.82 & 73.70 & 77.75 & 81.47 & 85.16 & 88.37 \\
\hline
\end{tabular}

Table 2. Zonal weather-yield models based on four alternative analyses.

\begin{tabular}{llcc}
\hline Types & \multicolumn{1}{c}{ Fitted Models } & R $^{2}$ & adj. R \\
\hline Model -1 & $\mathrm{Y}_{\text {est. }}=-31.65+0.74 \mathrm{Tr}+1.00 \mathrm{TMX}_{3}+0.35 \mathrm{TMX}_{5}-0.68 \mathrm{TMN}_{4}+0.01 \mathrm{ARF}_{2}+$ & 0.72 & 0.71 \\
& $0.06 \mathrm{ARF}_{9}+0.27 \mathrm{ARF}_{6}+0.24 \mathrm{TMN}_{6}$ & 0.65 & 1.77 \\
Model -2 & $\mathrm{Y}_{\text {est. }}=2.58+0.77 \mathrm{Tr}+1.09 \mathrm{PC}_{1}-0.90 \mathrm{PC}_{11}-0.69 \mathrm{PC}_{7}-0.48 \mathrm{PC}_{9}+0.46 \mathrm{PC}_{6}$ & 0.64 \\
Model -3 & $\mathrm{Y}_{\text {est. }}=7.20+1.93 \mathrm{CCIV}+0.07 \mathrm{ARF}_{8}-0.18 \mathrm{TMN}_{6}-0.17 \mathrm{SSH}_{7}-0.18 \mathrm{TMN}_{2}+$ & 0.92 & 0.91 \\
& $0.01 \mathrm{ARF}_{2}+0.01 \mathrm{ARF}_{11}-0.01 \mathrm{ARF}_{3}$ & 0.90 & 0.89 \\
Model -4 & $\mathrm{Y}_{\text {est. }}=11.07+9.63 \mathrm{D}_{5}+10.84 \mathrm{D}_{6}+7.47 \mathrm{D}_{4}+13.10 \mathrm{D}_{7}+5.55 \mathrm{D}_{3}+3.73 \mathrm{D}_{2}+$ & \multirow{2}{*}{1.19} \\
& $0.05 \mathrm{RF}_{8}-0.12 \mathrm{TMN}_{6}+1.47 \mathrm{D}_{1}-0.32 \mathrm{SSH}_{3}-0.05 \mathrm{RH}_{3}$ & \\
\hline
\end{tabular}

Model 1 - Weather parameters and trend yield as regressors; Model 2 - Principal component/weather scores and trend yield as regressors; Model 3 - Weather parameters and CCIV as regressors; Model 4 - Weather parameters and dummy variables as regressors; where, $\mathrm{Y}_{\text {est. }}$ - Model predicted yield (q/ha); Tr - Trend yield (q/ha); TMX - Av. maximum temperature; TMN Av. minimum temperature; ARF - Accumulated rainfall; RH - Av. relative humidity; SSH - Av. sunshine hours (1,2,3, $\ldots . ., 10 / 12$ refer to different fortnights); $\mathrm{PC}_{\mathrm{i}}-\mathrm{i}^{\text {th }}$ principal component score $(\mathrm{i}=1,2,3, \ldots \ldots \ldots)$; CCIV - Crop condition based indicator variable; $D_{i}-i^{\text {th }}$ dummy variable $(i=1,2, \ldots, 7$ i.e. $(8-1)$; SE - Standard error

subsets of weather variables were selected using a stepwise regression method (Draper and Smith, 1981) in which all variables were first included in the model and eliminated one at a time with decisions at any particular step conditioned by the result of the previous step. The predictive performance of the multiple linear regression based zonal weather-yield model-1 was decided on the basis of adj- $\mathrm{R}^{2}$, percent deviations of yield estimates from the real-time yields and root mean square errors (RMSEs).

In PC analysis, one third of the principal components of correlation matrix of the weather variables explained approximately $90 \%$ of the variation and the remaining components accounted for merely $10 \%$ of the total variation. Hence, the latter components were not considered to be of much practical significance. Principal component matrix alongwith variance explained by different PCs is depicted in Table 1. The crop yield models were obtained using weather based PC scores and linear time trend yield as regressors by following the step-wise regression method. Alternatively, the selected zonal model-2 was used to obtain the yield forecasts of all the districts under consideration. The zonal models- 1 and 2 described in Table 2 couldn't provide the satisfactory crop yield forecasting accuracy.

To further enhance the predictive performance, the weather based models were expanded by adding crop condition based categorical/indicator covariate (CCIV)/dummy regressors. The resulting models were labeled models-3 and 4 respectively. It was difficult to obtain the precise information on CCIV, which, we derived from the DOA crop yield data by following cluster analysis to formulate 3 to 8 non overlapping clusters respectively. Further, an indicator variable CCIV was generated by splitting the DOA crop yield series into eight non-overlapping classes corresponding to the yields $4-6,6-8,8-10,10-12,12-14,14-16,16-18$ and 18-20 q/ha, respectively thus seven dummies were prepared accordingly. Finally, the crop yield models were fitted by taking dummy variables along with 
Ravita and Urmil Verma / J. Appl. \& Nat. Sci. 9 (3): 1703- 1709 (2017)

Table 3. District-specific mustard yield estimates along with percent deviations from DOA yield (s) using four alternative models.

\begin{tabular}{|c|c|c|c|c|c|c|c|c|c|c|}
\hline \multirow[t]{2}{*}{ Districts } & \multirow[t]{2}{*}{ Year } & \multirow{2}{*}{$\begin{array}{c}\text { DOA } \\
\text { Yield } \\
\text { (q/ha) }\end{array}$} & \multicolumn{2}{|c|}{ Model-1 } & \multicolumn{2}{|c|}{ Model-2 } & \multicolumn{2}{|c|}{ Model-3 } & \multicolumn{2}{|c|}{ Model-4 } \\
\hline & & & $\begin{array}{c}\text { Fitted } \\
\text { yield } \\
(\mathrm{q} / \mathrm{ha})\end{array}$ & $\begin{array}{l}\text { RD } \\
\text { (\%) }\end{array}$ & $\begin{array}{l}\text { Fitted yield } \\
\text { (q/ha) }\end{array}$ & $\begin{array}{l}\text { RD } \\
(\%)\end{array}$ & $\begin{array}{l}\text { Fitted yield } \\
(\mathbf{q} / \mathbf{h a})\end{array}$ & $\begin{array}{l}\text { RD } \\
\text { (\%) }\end{array}$ & $\begin{array}{l}\text { Fitted yield } \\
\quad(\mathbf{q} / \mathrm{ha})\end{array}$ & $\begin{array}{l}\text { RD } \\
\text { (\%) }\end{array}$ \\
\hline \multirow[t]{5}{*}{ Hisar } & $2009-10$ & 19.30 & 13.56 & 29.75 & 15.20 & 21.22 & 15.88 & 17.72 & 16.40 & 15.02 \\
\hline & $10-11$ & 20.14 & 12.58 & 39.01 & 13.83 & 31.32 & 18.02 & 10.54 & 17.72 & 12.02 \\
\hline & $11-12$ & 17.07 & 13.93 & 18.37 & 14.38 & 15.76 & 15.76 & 7.67 & 15.39 & 9.82 \\
\hline & $12-13$ & 16.78 & 13.20 & 21.32 & 15.55 & 7.35 & 15.20 & 9.42 & 15.74 & 6.19 \\
\hline & $13-14$ & 16.26 & 11.78 & 27.57 & 14.59 & 10.26 & 14.74 & 9.32 & 15.13 & 6.94 \\
\hline \multirow[t]{5}{*}{ Bhiwani } & 09-10 & 15.41 & 13.42 & 12.93 & 15.06 & 2.28 & 15.88 & -3.05 & 16.40 & -6.43 \\
\hline & $10-11$ & 17.27 & 12.15 & 29.65 & 13.69 & 20.71 & 18.02 & -4.33 & 17.2 & -2.60 \\
\hline & $11-12$ & 14.00 & 13.82 & 1.32 & 14.26 & -1.83 & 15.76 & -12.57 & 15.39 & -9.95 \\
\hline & $12-13$ & 16.40 & 13.09 & 20.18 & 15.43 & 5.91 & 15.20 & 7.32 & 15.74 & 4.02 \\
\hline & $13-14$ & 15.16 & 11.68 & 22.95 & 14.49 & 4.41 & 14.74 & -2.74 & 15.13 & 0.19 \\
\hline \multirow[t]{5}{*}{ Sirsa } & $09-10$ & 17.81 & 13.72 & 22.96 & 15.37 & 13.68 & 15.88 & 10.84 & 16.40 & 7.91 \\
\hline & $10-11$ & 19.82 & 12.46 & 37.13 & 14.02 & 29.28 & 18.02 & 9.09 & 17.72 & 10.60 \\
\hline & $11-12$ & 16.78 & 14.14 & 15.73 & 14.59 & 13.02 & 15.76 & 6.08 & 15.39 & 8.26 \\
\hline & $12-13$ & 16.47 & 13.42 & 18.49 & 15.78 & 4.20 & 15.20 & 7.72 & 15.74 & 4.43 \\
\hline & $13-14$ & 17.37 & 12.03 & 30.75 & 14.85 & 14.49 & 14.74 & 15.12 & 15.13 & 12.89 \\
\hline \multirow[t]{5}{*}{$\mathrm{F} / \mathrm{bad}$} & $09-10$ & 19.08 & 14.12 & 25.99 & 15.79 & 17.24 & 15.88 & 16.77 & 16.40 & 14.04 \\
\hline & $10-11$ & 21.26 & 12.84 & 39.58 & 14.42 & 32.19 & 18.02 & 15.25 & 17.72 & 16.66 \\
\hline & $11-12$ & 18.66 & 14.50 & 22.32 & 14.96 & 19.81 & 15.76 & 15.54 & 15.39 & 17.51 \\
\hline & $12-13$ & 15.99 & 13.76 & 13.92 & 16.13 & -0.89 & 15.20 & 4.95 & 14.74 & 1.56 \\
\hline & $13-14$ & 18.53 & 12.34 & 33.41 & 15.18 & 18.10 & 16.68 & 10.01 & 16.34 & 11.82 \\
\hline \multirow[t]{5}{*}{ M/garh } & $09-10$ & 15.41 & 14.13 & 8.32 & 15.80 & -2.51 & 15.88 & -3.05 & 16.40 & -6.43 \\
\hline & $10-11$ & 19.02 & 12.86 & 32.39 & 14.43 & 24.12 & 18.02 & 5.27 & 17.72 & 6.84 \\
\hline & $11-12$ & 18.27 & 14.53 & 20.45 & 15.00 & 17.28 & 15.76 & 13.74 & 15.39 & 15.74 \\
\hline & $12-13$ & 16.99 & 13.81 & 18.72 & 16.18 & 4.78 & 15.20 & 10.54 & 15.74 & 7.35 \\
\hline & $13-14$ & 16.99 & 12.40 & 27.02 & 15.24 & 10.31 & 16.68 & 1.85 & 16.34 & 3.83 \\
\hline \multirow[t]{5}{*}{ Rewari } & $09-10$ & 13.84 & 15.30 & -10.53 & 17.01 & -22.93 & 17.81 & -28.69 & 17.61 & -27.24 \\
\hline & $10-11$ & 19.79 & 14.03 & 29.11 & 15.65 & 20.93 & 19.95 & -0.80 & 18.93 & 4.36 \\
\hline & $11-12$ & 18.40 & 15.69 & 14.74 & 16.20 & 11.94 & 17.69 & 3.85 & 16.60 & 9.77 \\
\hline & $12-13$ & 21.57 & 14.96 & 30.66 & 17.37 & 19.46 & 17.13 & 20.59 & 16.95 & 21.43 \\
\hline & $13-14$ & 18.64 & 13.54 & 27.37 & 16.42 & 11.89 & 16.68 & 10.54 & 16.34 & 12.34 \\
\hline \multirow[t]{5}{*}{ Jhajjar } & $09-10$ & 15.97 & 13.49 & 15.52 & 15.14 & 5.23 & 15.88 & 0.56 & 16.40 & -2.70 \\
\hline & $10-11$ & 17.95 & 12.27 & 31.66 & 13.82 & 23.03 & 18.09 & -0.38 & 17.72 & 1.29 \\
\hline & $11-12$ & 15.95 & 13.97 & 12.41 & 14.42 & 9.61 & 15.76 & 1.19 & 15.39 & 3.49 \\
\hline & $12-13$ & 16.86 & 13.29 & 21.17 & 15.64 & 7.24 & 15.20 & 9.85 & 15.74 & 6.64 \\
\hline & $13-14$ & 15.49 & 11.92 & 23.02 & 14.74 & 4.81 & 14.74 & 4.81 & 15.13 & 2.31 \\
\hline \multirow[t]{5}{*}{ Gurgaon } & $09-10$ & 17.83 & 13.38 & 24.95 & 15.02 & 15.76 & 15.88 & 10.94 & 16.40 & 8.01 \\
\hline & $10-11$ & 18.68 & 12.08 & 35.36 & 13.62 & 27.11 & 18.02 & 3.54 & 17.72 & 5.15 \\
\hline & $11-12$ & 20.25 & 13.71 & 32.29 & 14.15 & 30.13 & 15.76 & 22.17 & 15.39 & 23.98 \\
\hline & $12-13$ & 20.26 & 12.96 & 36.04 & 15.29 & 24.52 & 15.20 & 24.98 & 15.74 & 22.31 \\
\hline & $13-14$ & 15.94 & 11.52 & 27.74 & 14.32 & 10.15 & 14.74 & 7.50 & 15.13 & 5.07 \\
\hline
\end{tabular}

weather input as regressors. Model predicted yield of all the districts along with observed yield and percent deviations from DOA yield(s) based on alternative analyses are given in Table 3.

Five-steps ahead (out-of-model development period i.e. 2009-10, 2010-11, 2011-12, 2012-13 and 2013-14) district-level mustard yield estimates (Table 3) were obtained on the basis of above models. A comparative view in terms of percent deviations of the forecast yield(s) from DOA yield estimates and district-level RMSEs for the post-sample period(s) are given in Tables 4 and 5.

Thus, it has been observed that the regression equations with an apparently desirable degree of fit, as measured by the coefficient of multiple correlation $\mathrm{R}^{2}$, but with a low value of the Durbin-Watson statistic, couldn't provide the adequate predictive accuracy. However, the objective of mustard yield modeling was to assess the predictive accuracies of the developed models for estimating crop yields and how the accuracies are influenced by different statistical procedures. The yield(s) estimated by zonal weather-yield models 1 and 2 had sometimes wide percent deviations from the observed yields i.e. too high than considered to be acceptable for reliable yield prediction purposes. Consequent upon, an attempt was made to improve the predictive accuracy of the zonal models by identifying and adding crop condition based categorical covariate/ dummy regressors. In particular, adding the CCIV to the zonal weather-yield models 
Ravita and Urmil Verma / J. Appl. \& Nat. Sci. 9 (3): 1703- 1709 (2017)

Table 4. A comparative view in terms of average absolute percent deviations based on the alternative models.

\begin{tabular}{|c|c|c|c|c|}
\hline Districts & Model-1 & Model-2 & Model-3 & Model-4 \\
\hline Hisar & 27.20 & 17.18 & 10.93 & 10.00 \\
\hline Bhiwani & 17.41 & 7.03 & 6.00 & 4.64 \\
\hline Sirsa & 25.01 & 14.93 & 9.77 & 8.82 \\
\hline Fatehabad & 27.04 & 17.65 & 12.50 & 12.32 \\
\hline Mahendergarh & 21.38 & 11.80 & 6.89 & 8.04 \\
\hline Rewari & 22.48 & 17.43 & 12.89 & 15.03 \\
\hline Jhajjar & 20.76 & 9.98 & 3.36 & 3.29 \\
\hline Gurgaon & 31.28 & 21.53 & 13.83 & 12.90 \\
\hline
\end{tabular}

Table 5. District-level RMSEs for the post-sample period.

\begin{tabular}{lcccc}
\hline Districts/model (s) & Model-1 & Model-2 & Model-3 & Model-4 \\
\hline Hisar & 5.24 & 3.69 & 2.13 & 1.97 \\
Bhiwani & 3.26 & 1.69 & 1.05 & 0.84 \\
Sirsa & 4.81 & 3.20 & 1.82 & 1.67 \\
Fatehabad & 5.59 & 4.06 & 2.58 & 2.65 \\
Mahendergarh & 4.12 & 2.67 & 1.47 & 1.61 \\
Rewari & 4.74 & 3.30 & 2.82 & 2.99 \\
Jhajjar & 3.68 & 2.11 & 0.82 & 0.62 \\
Gurgaon & 5.98 & 4.43 & 3.21 & 3.09 \\
\hline
\end{tabular}

substantially improved the predictive accuracy and produced quite satisfactory district-level mustard yield estimates. The results achieved are in agreement with the study conducted by Verma et al. (2011), where the use of weather parameters along with time trend couldn't serve the purpose of yield prediction with desired accuracy for cotton and mustard crops in Haryana. Further, it was felt that the models could be strengthened by adding some agronomic/ biometrical parameters for yield forecasting purpose. Needless to emphasize that incorporating the crop condition term as dummy variable with weather parameters provided significant improvement in the prediction capability of zonal models for district-level yield prediction in the state.

Hence, based on this empirical study, using CCIV as an indicator variable is recommended to incorporate with weather parameters to enhance the predictive accuracy of the zonal weather-yield models. The percent deviations falling within tolerable limits favor the use of zonal models 3 and 4 for district-level pre-harvest mustard yield forecasing in Haryana.

\section{Conclusion}

The zonal weather-yield models have been developed using multiple linear regression, principal component and cluster analyses to achieve the rapeseed-mustard yield estimation in major mustard growing districts viz., Hisar, Bhiwani, Sirsa, Fatehabad, Mahendergarh, Rewari, Jhajjar and Gurgaon districts of Haryana. The predictive performance(s) of the contending models were observed in terms of the percent deviations of mustard yield forecasts in relation to the observed yield(s) and RMSEs as well. Zonal yield models incorporating CCIV and weather variables consistently showed the satisfactory results in capturing the percent relative deviations and performed well with lower error metrics as compared to the remaining models in all time regimes. The estimated yield(s) from the selected zonal models indicated good agreement with DOA mustard yields by showing 5-10 percent deviations in most of the districts however for twothree districts, it gave 12-13 percent deviations possibly due to the smaller set of data available for those districts.

\section{ACKNOWLEDGEMENTS}

The weather data received from Haryana Space Applications Centre, Hisar and Department of Agrometeorology, CCS HAU, Hisar, India are gratefully acknowledged.

\section{REFERENCES}

Adrian, D. (2012). A model-based approach to forecasting corn and soybean yields. USDA, National Agricultural Statistics Service, Research \& Development Division.

Ahmad, T. and Kathuria, O.P. (2010). Estimation of crop yield at block level. Adv. Appl. Res. 2(2), 164-172.

Andarzian, B., Bakhshandeh, A.M., Bannayan, M., Emam, Y., Fathi, G. and Alami, S. (2008). Wheat Pot : a simple model for spring wheat Yp using monthly weather data. Biosyst. Eng. 99, 487-495.

Azfar, M., Sisodia, B. V. S., Rai, V. N. and Devi, M. (2015). Pre-harvest forecast models for rapeseed \& mustard yield using principal component analysis of weather variables, Mausam 66(4), 761-766.

Dadhwal, V. K., Sehgal, V. K., Singh, R. P. and Rajak, D. R. 
(2003). Wheat yield modeling using satellite remote sensing with weather data; recent Indian experience. Mausam 54, 253-262.

Draper, N. and Smith, H. (1981). Applied Regression Analysis. 2nd edition. New York, Wiley.

Kaiser, H.F. (1960). The application of electronic computers to factor analysis. Educational and Psychological Measurement 20, 141-151.

Kandiannan, K., Chandaragiri, K. K., Sankaran, N., Balasubramanian, T. N. and Kailasam, C. (2002). Cropweather model for turmeric yield forecasting for Coimbatore District, Tamil Nadu, India, Agricultural and Forest Meteorology 112, 133-137.

Shabnam, Bansal, S. K. and Dabas, D. S. (2013). Use of time -series data of temperature and yield to assess the impact of climate change on crop yield using mustard in Haryana, International Research Journal of Social Sci- ences 2(4), 31-33.

Shaw P.J.A. (2003). Multivariate statistics for the Environmental Sciences, Hodder-arnold ISBN 0-3408-0763-6.

Field, A. (2000). Discovering Statistics using SPSS for Windows, London - Thousand Oaks - New Delhi, Sage publications.

Goyal, M. and Verma, U. (2015). Development of weatherspectral models for pre-harvest wheat yield prediction on agro-climatic zone basis in Haryana, International $J$. of Agricultural and Statistical Sciences 11(1), 73-79.

Verma, U., Dabas, D. S. and Singh, J. P. (2011). Regression, multivariate techniques, time series and mixed modelling in context of pre-harvest wheat, mustard, cotton and sugarcane yield prediction in Haryana using the SAS system, Research Bulletin, Department of Soil Science, CCS Haryana Agricultural University, Hisar, Haryana, pp.1-154, 